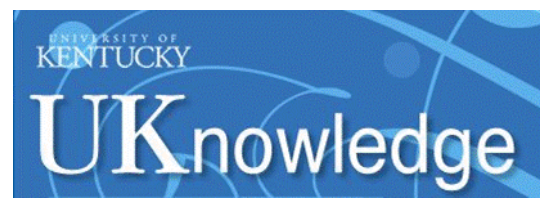

University of Kentucky

UKnowledge

\title{
Temperature Variations from Hubble Space Telescope Imagery and Spectroscopy of NGC 7009
}

\author{
R. H. Rubin \\ University College London, UK \\ N. J. Bhatt \\ NASA/Ames Research Centre \\ R. J. Dufour \\ Rice University \\ B. A. Buckalew \\ Rice University \\ M. J. Barlow \\ University College London, UK
}

See next page for additional authors

Follow this and additional works at: https://uknowledge.uky.edu/physastron_facpub

Part of the Astrophysics and Astronomy Commons, and the Physics Commons

Right click to open a feedback form in a new tab to let us know how this document benefits you.

\section{Repository Citation}

Rubin, R. H.; Bhatt, N. J.; Dufour, R. J.; Buckalew, B. A.; Barlow, M. J.; Liu, X.-W.; Storey, P. J.; Balick, B.; Ferland, Gary J.; Harrington, J. P.; and Martin, P. G., "Temperature Variations from Hubble Space Telescope Imagery and Spectroscopy of NGC 7009" (2002). Physics and Astronomy Faculty Publications. 47. https://uknowledge.uky.edu/physastron_facpub/47

This Article is brought to you for free and open access by the Physics and Astronomy at UKnowledge. It has been accepted for inclusion in Physics and Astronomy Faculty Publications by an authorized administrator of UKnowledge. For more information, please contact UKnowledge@lsv.uky.edu. 


\title{
Temperature Variations from Hubble Space Telescope Imagery and Spectroscopy of NGC 7009
}

\author{
Digital Object Identifier (DOI) \\ http://dx.doi.org/10.1046/j.1365-8711.2002.05567.x
}

\section{Notes/Citation Information}

Published in Monthly Notices of the Royal Astronomical Society, v. 334, issue 4, p. 777-786.

This article has been accepted for publication in Monthly Notices of the Royal Astronomical Society (c): 2002 The Authors Published by Oxford University Press on behalf of the Royal Astronomical Society. All rights reserved.

The copyright holder has granted the permission for posting the article here.

\section{Authors}

R. H. Rubin, N. J. Bhatt, R. J. Dufour, B. A. Buckalew, M. J. Barlow, X.-W. Liu, P. J. Storey, B. Balick, Gary J. Ferland, J. P. Harrington, and P. G. Martin 


\title{
Temperature variations from Hubble Space Telescope imagery and spectroscopy of NGC 7009
}

\author{
R. H. Rubin, ${ }^{1,2 \star}$ N. J. Bhatt, ${ }^{1}$ R. J. Dufour, ${ }^{3}$ B. A. Buckalew, ${ }^{3}$ \\ M. J. Barlow, ${ }^{4}$ X.-W. Liu, ${ }^{4}$ P. J. Storey, ${ }^{4}$ B. Balick, ${ }^{5}$ G. J. Ferland, ${ }^{6}$ \\ J. P. Harrington ${ }^{7}$ and P. G. Martin 8 \\ ${ }^{1}$ NASA/Ames Research Centre, Moffett Field, CA 94035-1000, USA \\ ${ }^{2}$ Orion Enterprises, M. S. 245-6, Moffett Field, CA 94035-1000, USA \\ ${ }^{3}$ Physics \& Astronomy Department, Rice University, MS 61, Houston, TX 77005-1892, USA \\ ${ }^{4}$ Physics \& Astronomy Department, University College London, Gower Street, London, WC1E 6BT \\ ${ }^{5}$ Astronomy Department, University of Washington, Seattle, WA 98195-1580, USA \\ ${ }^{6}$ Physics \& Astronomy Department, University of Kentucky, Lexington, KY 40506-0055, USA \\ ${ }^{7}$ Astronomy Department, University of Maryland, College Park, MD 20742-2421, USA \\ ${ }^{8}$ Canadian Institute for Theoretical Astrophysics, University of Toronto, Toronto, ON M5S 3H8, Canada
}

Accepted 2002 April 3. Received 2002 March 1; in original form 2001 December 13

\begin{abstract}
We present new Hubble Space Telescope (HST)/WFPC2 imagery and STIS long-slit spectroscopy of the planetary nebula NGC 7009. The primary goal was to obtain high spatial resolution of the intrinsic line ratio [O III] 4364/5008 and thereby evaluate the electron temperature $\left(T_{\mathrm{e}}\right)$ and the fractional mean-square $T_{\mathrm{e}}$ variation $\left(t_{A}^{2}\right)$ across the nebula. The WFPC2 $T_{\mathrm{e}}$ map is rather uniform; almost all values are between $9000-11000 \mathrm{~K}$, with the higher $T_{\mathrm{e}}$ values closely coinciding with the inner $\mathrm{He}^{++}$zone. The results indicate very small values $\lesssim 0.01$ - for $t_{A}^{2}$ throughout. Our STIS data allow an even more direct determination of $T_{\mathrm{e}}$ and $t_{A}^{2}$, albeit for a much smaller area than with WFPC2. We present results from binning the data along the slit into tiles that are 0.5 -arcsec square (matching the slit width). The average [O III] temperature using 45 tiles (excluding the central star and STIS fiducial bars) is $10139 \mathrm{~K}$; $t_{A}^{2}$ is 0.0035 . The measurements of $T_{\mathrm{e}}$ reported here are an average along each line of sight. Therefore, despite finding remarkably low $t_{A}^{2}$, we cannot completely rule out temperature fluctuations along the line of sight as the cause of the large abundance discrepancy between heavy element abundances inferred from collisionally excited emission lines compared to those derived from recombination lines.
\end{abstract}

Key words: ISM: abundances - ISM: atoms - ISM: individual: NGC 7009-planetary nebulae: general.

\section{INTRODUCTION}

Most observational tests of the chemical evolution of the Universe rest on emission line objects; these define the endpoints of stellar evolution and probe the current state of the interstellar medium. Gaseous nebulae are laboratories for understanding physical processes in all emission-line sources, and probes for stellar, galactic and primordial nucleosynthesis.

There is a fundamental issue that continues to be problematic - the discrepancy between heavy element abundances inferred from emission lines that are collisionally excited compared with those due to recombination/cascading, the so-called 'recombination lines'. Stud-

^E-mail: rubin@cygnus.arc.nasa.gov ies of planetary nebulae (PNs) contrasting recombination and collisional abundances (Liu et al. 1995; Kwitter \& Henry 1998) often find differences exceeding a factor of 2 . In an extensive study of NGC 6153, Liu et al. (2000) found that $\mathrm{C}^{++} / \mathrm{H}^{+}, \mathrm{N}^{++} / \mathrm{H}^{+}, \mathrm{O}^{++} / \mathrm{H}^{+}$ and $\mathrm{Ne}^{++} / \mathrm{H}^{+}$ratios derived from optical recombination lines are all a factor of $\sim 10$ higher than the corresponding values deduced from collisionally-excited lines. Abundances determined from these two methods disagree by a factor larger than the spread of abundances used to determine such fundamental quantities as Galactic abundance gradients (e.g. Shaver et al. 1983; Simpson et al. 1995; Henry $\&$ Worthey 1999).

Most of the efforts to explain the abundance puzzle between collisional and recombination values have attempted to do so by examining electron temperature $\left(T_{\mathrm{e}}\right)$ variations in the plasma. This is often done, using the formalism of Peimbert (1967), in terms of the 
[fractional] mean-square variation $\left(t^{2}\right)$ of $T_{\mathrm{e}}$. The inferred metallicity obtained by using the usual (optical/UV) forbidden lines is very sensitive to $T_{\mathrm{e}}$ (exponential) and $t^{2}$. On the other hand, recombination lines are rather insensitive to $T_{\mathrm{e}}$ and $t^{2}$. Agreement close to the higher recombination value can be forced in the derived abundance by attributing the difference to (solving for) $t^{2}$. Let us consider the case of the PN NGC 7009, also known as the 'Saturn Nebula'; its image produced by Balick et al. (1998) from WFPC2 data has graced the cover of coffee-table books (Petersen \& Brandt 1998). NGC 7009 has stood near the centre of the abundances controversy. It has long been known that its recombination lines are very strong, particularly those of O II. Liu et al. (1995) found that the recombination $\mathrm{C}, \mathrm{N}$ and $\mathrm{O}$ abundances are a factor of $\sim 5$ larger than the corresponding collisional abundances. This was recently found to be the case also for neon (Luo, Liu \& Barlow 2001). One possible reconciliation of these two drastically different abundances, which Liu et al. (1995) discuss, is to invoke large $t^{2}$. For oxygen in NGC 7009 , by invoking $t^{2} \sim 0.1$, agreement can be forced in the abundance derived from optical lines close to the higher recombination value - a value more than 2.5 times larger than the solar $\mathrm{O} / \mathrm{H}$ of $7.41 \times 10^{-4}$ (Grevesse \& Sauval 1998). Such a large $t^{2}$ is not at all predicted by current theory/models (e.g. Kingdon \& Ferland 1998).

The current unsettled situation has led to efforts to broaden the study to include other variables besides $T_{\mathrm{e}}$ to analyse the effects upon abundance determinations. One promising avenue is to examine this considering density variations, abundance variations and $T_{\mathrm{e}}$ variations in combination. Liu et al. (2000) took this approach in their investigation of the PN NGC 6153 with a two-phase empirical model. Recall this object has even more disparate abundance differences (factor of $\sim 10$ ) than NGC 7009 does. Péquignot et al. (2002) continued the study using photoionization models including two components with different heavy element abundances. Basically, one phase is small, dense, ionized clumps that are highly enhanced in heavy elements and deficient in hydrogen. This phase would have $T_{\mathrm{e}} \sim 10^{3} \mathrm{~K}$ and contribute almost all the heavy element recombination line emission. These clumps are embedded in the second phase, which has the more usual nebular properties/composition, with $T_{\mathrm{e}} \sim 10^{4} \mathrm{~K}$, and which is responsible for almost all the emission in the optical/UV collisionally excited lines. The latter phase comprises the great bulk of the nebular mass. There is ongoing work by this same group to try to explain other PNs of this kind, including the Galactic bulge PN M 1-42, which has a factor of $~ 20$ abundance dichotomy (Liu et al. 2001) and Hf 2-2, which has the most extreme abundance difference to date, a factor of $\sim 80$ (Liu 2002).

In this paper, we focus solely on $T_{\mathrm{e}}$ variations with the purpose to test whether the observational data support the possibility that $t^{2}$ in NGC 7009 may be as large as $\sim 0.1$. In Section 2, we present the HST observations and data reduction procedures. Section 3 describes our techniques for obtaining emission line fluxes from the WFPC2 data and how we use cospatial STIS data to help this process. In Section 4, there is a discussion and analysis of extinction. We determine the electron temperature distributions in Section 5. In Section 6, we analyse the $T_{\mathrm{e}}$ distributions in terms of average temperatures and fractional mean-square temperature variations in the plane of the sky. Section 7 provides a discussion and conclusions.

\section{HST OBSERVATIONS AND DATA REDUCTION}

The observations of NGC 7009 described here were taken as part of our HST Cycle 8 program GO-8114. For both the WFPC2 and STIS visits, we set our positions with respect to the position of the central star (CS): $\alpha, \delta=21^{\mathrm{h}} 04^{\mathrm{m}} 10^{\mathrm{s}} .81,-11^{\circ} 21^{\prime} 47^{\prime \prime} .91$ ) (equinox J2000).

\subsection{WFPC2 observations}

We observed with WFPC2 on 2000 April 6-7 (UT) as the first visit of the GO-8114 program. Observations (with corresponding line of interest) of NGC 7009 were made in line filters F437N ([O III] $4364 \AA$ ) , F487N (H $\beta 4863 \AA$ ), F502N ([O III] $5008 \AA$ ) and F656N (H $\alpha 6565 \AA$ ), plus continuum filter F547M. All wavelengths in this paper are vacuum rest wavelengths. The images were placed in the WF3 chip (with the CS at WF3-FIX). The pixel size is 0.0996 arcsec. In order to clean cosmic rays, two images were taken in each filter except for three images in F437N. Total integration times were, respectively, 2200, 520, 320, 400 and $240 \mathrm{~s}$.

The data sets we processed (also for STIS) were those obtained after sufficient time elapsed from the observation dates in order that 'best reference files' would be stable/finalized. We requested On-the-Fly Calibration for science files and best reference files. We then co-added and cosmic ray cleaned images using standard packages in IRAF. ${ }^{1}$ Flux calibration for each filter was carried out by basically following the procedures used for the WFPC2 Exposure Time Calculator. These account for the integration time, analogueto-digital converter gain of 7 (used for F437N and F487N) or 14 electron $\mathrm{DN}^{-1}$ (used for F502N, F547M and F656N; DN stands for 'Data Number'), and the total system throughput.

The calculations for the contributions to the narrow-band filter observations of the continuum and other lines will be discussed later (Section 3) after the STIS observations section.

\subsection{STIS observations}

On 2000 August 9 (UT), STIS long-slit spectra of NGC 7009 were taken. These data comprise 3 orbits and were taken at position angle $(\mathrm{PA})=86.72^{\circ}$. Our original plan was to align the slit with the major axis of NGC 7009, which is at PA $=78.5^{\circ}$. However, we were advised by STScI that there were no guide star pairs for our planned visits. We settled on the revised PA, a change of only $8.22^{\circ}$, as this was the closest to the major axis that allowed for a pair of guide stars. We used a slit width of 0.5 arcsec and a slit length of 52 arcsec for these CCD observations.

Because the CS in NGC 7009 is reasonably bright $(V=12.78$, $B=12.66$ ), it would have caused problems (bleeding, saturation, etc.), especially on long exposures, if it were in our science slit. On the other hand, the CS provides an excellent fiducial point in the nebula, where we may assess the positional registration of emission lines in our various exposures with STIS. Thus, we observed with the CS off the upper edge of our $52 \times 0.5$ arcsec slit by 0.1 arcsec. In other words, our slit is defined with the CS moved 0.35 arcsec $(0.25+0.1)$ in the dispersion direction $(\sim \mathrm{NNW})$. Furthermore, the slit was not centred on the CS in the spatial direction. Because the structure on the west (W) side of the nebula was of more interest to us, we displaced the slit centre 3.5 arcsec from the CS in the spatial direction $\left(\sim \mathrm{W}\right.$ along $\left.\mathrm{PA}=86.72^{\circ}\right)$. Spectra were taken with gratings G430M (with wavelength settings: 3680, 4194, 4451, 4706, 4961) and G750M (settings: 5734, 6581). Each exposure was done in accumulation mode and at least two spectra were taken at each setting in order to cosmic ray (CR) clean. After retrieving the

\footnotetext{
${ }^{1}$ IRAF is distributed by NOAO, which is operated by AURA, under cooper-
} ative agreement with NSF. 
data sets, we then co-added and cosmic ray cleaned images using standard packages in IRAF. Calibrations to produce two-dimensional (2D) rectified images were then carried out. From these, we singled out specific emission lines for further investigation.

Data for the [O III] 4364- $\AA$ line as well as $\mathrm{H} \gamma 4342 \AA$ were contained in the $\mathrm{G} 430 \mathrm{M} / 4451$ grating setting $(150 \mathrm{~s})$; for the [O III] 5008-, 4960- $\AA$ lines and $\mathrm{H} \beta$ 4863- $\AA$ lines, G430M/4961 was used (80 s); for $\mathrm{H} \alpha 6565 \AA$ and the [N II] 6550-, 6585- $\AA$ lines, G750M/ 6581 was used $(90 \mathrm{~s})$. For G430M, the dispersion is $0.28 \AA \mathrm{pixel}^{-1}$ for a point source and the plate scale $0.05 \operatorname{arcsec}_{\text {pixel }}^{-1}$ (Leitherer et al. 2001, Chapter 13). For a uniformly filled slit with width 0.5 arcsec, a degradation in resolving power by a factor of 10 is expected to a spectral resolution of $2.8 \AA$. For G750M, the dispersion is $0.56 \AA$ pixel $^{-1}$ for a point source and the plate scale 0.05 arcsec pixel $^{-1}$. For a uniformly filled slit with width 0.5 arcsec, the spectral resolution would be $5.6 \AA$. For the analysis presented here, we were interested mainly in the distribution of line flux along the slit spatial direction. This was accomplished with the IRAF routine BLKAVG in conjunction with specialized software tools that we developed ourselves.

Even after applying the standard CR rejection there still remain many bad pixels due to CRs and/or hot pixels. There is considerable danger that including these can corrupt the flux values we seek. The program developed to eliminate these remaining bad pixels is called PIXHUNTER, which is described briefly in Appendix A. Once the columns containing the line have been cleaned for deviant pixels, we are ready to subtract an equivalent spectral range of continuum. We do this by using IRAF functions, including BLKAVG, to operate upon the appropriate sections of cleaned continuum. The one-dimensional (1D) distribution of line flux versus spatial coordinate for the various emission lines of interest is what we need for our subsequent physical analysis.

We note that there is excellent agreement with a cross check of the $1 \mathrm{D}$ results of flux versus spatial direction by comparing with 1D results of flux versus wavelength for a corresponding spatial sample. The latter were measured with the SPLOT package. With this, the underlying continuum is fitted and the integrated line flux determined with the $e$-option (area under the line profile), which was preferable to fitting with a Gaussian profile. Because of the spectral impurity introduced by the relatively wide slit used, the line profiles have flatter tops and less extended bases (i.e. they are more 'trapezoidal') than the Gaussian fits. It is also apparent that the Gaussian fit is overestimating the line flux.

Both the [O III] 5008- and 4960-Å lines were observed simultaneously with the G430M/4961 grating setting. We desired to accurately measure the ratio of these line fluxes to compare with the most recent calculation for the theoretical intensity ratio 2.984 (Storey \& Zeippen 2000). Because both transitions arise from the same upper level, the intrinsic flux ratio depends only on the transition probabilities ( $A$-values) and wavelengths. What we found was a surprising variation in the $F(5008) / F(4960)$ ratio with position along the slit. This amounts to a variation in the ratio of roughly $3.0 \pm 0.1$. Furthermore, it appears more-or-less periodic with an $\sim 3.5$-arcsec cycle.

According to Ted Gull (private communication), this is an instrumental effect and is a ratio of two fringe patterns. The source of the problem is a thin blocker filter that had to be matched with each grating and the best (and only) location that it could be placed was above the grating in a stable mounting. There is some discussion of fringing at the STIS web site (http://www.stsci.edu/instruments/ stis/performance/anomalies). More specific information appears in chapter 7 of the STIS Instrument Handbook (Leitherer et al. 2001) under a section called 'Fringing due to the Order Sorter Fil- ters'. (See www.stsci.edu/instruments/stis/documents/handbooks/ cycle11/c07_performance2.html\#326437.) This distinct fringing pattern also appears in similar STIS data of the Orion Nebula obtained under program GO-7514 (PI RR).

To attempt to do anything about fringing would probably require a dedicated $H S T$ /STIS calibration program. We do not treat this further here. As will be seen, the results for the temperature analyses are in good accord between the two data sets using STIS and WFPC2, which provides support that any fringing in the STIS data is not affecting our conclusions. If there were fringing in the $F(5008)$ / $F(4364)$ ratio at the same level as for the $F(5008) / F(4960)$ ratio, the \pm 3.3 per cent error would result in only a minor $T_{\mathrm{e}}$ error, e.g. $\pm 100 \mathrm{~K}$ at $T_{\mathrm{e}}=10^{4} \mathrm{~K}$ (see Section 5). Because of the fringe pattern, we measured the fluxes $F(5008)$ and $F(4960)$ over an integer number of fringing cycles and then calculated the $F(5008) / F(4960)$ ratio. The overall value is 3.008 . When we apply a very small differential extinction correction (see Section 4), the intrinsic ratio becomes 3.000 , which is in good agreement with the theoretical prediction.

\section{EXTRACTION OF EMISSION LINE FLUXES FROM WFPC2 DATA}

One of the most important operations involved partitioning the contributions to the $\mathrm{F} 437 \mathrm{~N}$ emission. We consider these as due to [O III] $4364, \mathrm{H} \gamma$ and continuum emission. (An examination of our STIS spectra shows no other emission lines will contribute significant flux.) It is necessary to take into account the total system throughput for the F437N filter (Biretta et al. 2001). A finer scale digital version of the total system throughput for this or any WFPC2 filter can also be produced using the task CALCBAND. We used a digital form to obtain the throughputs for both the [O III] 4364 and $\mathrm{H} \gamma$ lines accounting for the Doppler correction for the actual velocity of NGC 7009 at the time of observation. This was $-20.71 \mathrm{~km} \mathrm{~s}^{-1}$ after accounting for the heliocentric velocity of 7009 , which is $-46.6 \mathrm{~km} \mathrm{~s}^{-1}$ (Schneider et al. 1983). While still a net blueshift $(-0.3 \AA)$, this does result in a slightly higher $\mathrm{H} \gamma$ leakage due to near maximum redshift of the Earth's motion at the time of observation. The relative transmission at the Doppler corrected wavelengths for $\mathrm{H} \gamma$ to 4364 was 0.0253 .

In order to assess the contribution of 4364 and $\mathrm{H} \gamma$ leakage to our F437N data, we did a careful spatial registration/comparison of the highest signal-to-noise ratio (S/N) portions of our STIS long-slit data with corresponding WFPC2 filters. Two areas were chosen that we call $\mathrm{E} 1$ and $\mathrm{WW}$ that were regions of higher $\mathrm{S} / \mathrm{N}$ and avoided the $\mathrm{CS}$ as well as the fiducial bars. They span from 1.05 to 7.60 arcsec east and from 0.75 to 8.10 arcsec west of the CS, respectively, along our 0.5 arcsec-wide STIS slit. The registration is very close with the E1 area from STIS 3.275 and from WFPC2 $3.2737 \mathrm{arcsec}^{2}$. For WW, areas are, respectively, 3.675 and $3.6705 \operatorname{arcsec}^{2}$.

Initially, we also tried to determine the continuum contribution to F437N from the spatial registration of our STIS data with the corresponding WFPC2 filters. All our efforts resulted in continuum fluxes that were generally too large for what could possibly fit for the WFPC2 observations. While it is not clear why the flux levels underlying the emission lines (not just 4364 but also $\mathrm{H} \alpha, \mathrm{H} \beta$ and 5008 ) were invariably too high, the integration times with STIS were insufficient to provide adequate signal-to-noise ratios to measure these continuum fluxes.

Because the dominant contribution to the nebular continuum for NGC 7009 is recombination processes, we estimate the continuum contribution to each of the WFPC2 filters from recombination theory. These were provided independently by two of us (JPH 
and GJF) from theoretical continuum models, with similar results. For these, we assumed $T_{\mathrm{e}}=10000 \mathrm{~K}, \mathrm{~N}(\mathrm{He}) / \mathrm{N}(\mathrm{H})=0.11$ and ionic fractions: $\mathrm{N}\left(\mathrm{He}^{+}\right) / \mathrm{N}(\mathrm{He})=0.88$ and $\mathrm{N}\left(\mathrm{He}^{++}\right) / \mathrm{N}(\mathrm{He})=0.12$, which are essentially the NGC 7009 values in Kingsburgh \& Barlow (1994). The scaled values adopted for the continuum emission relative to the $\mathrm{H} \beta$ line emission (all intrinsic) are $6.91 \times 10^{-4}$, $6.53 \times 10^{-4}, 6.45 \times 10^{-4}$ and $5.65 \times 10^{-4} \AA^{-1}$ for F437N, F487N, F502N and F656N, respectively. To obtain the total continuum contribution in the respective filters, one multiplies by the appropriate 'rectwidth' (RECTW) 31.994, 33.8, 35.562 and 29.89 Å obtained using the task BANDPAR. ${ }^{2}$

This provides the predicted continuum correction for $\mathrm{F} 487 \mathrm{~N}$ of $0.02207 I(\mathrm{H} \beta)$, where $I(\mathrm{H} \beta)$ is the intrinsic flux. Because both line and continuum are equally affected by extinction, we may also work with the observed fluxes without regard to extinction correction here. To predict the continuum correction for F656N and F437N, we scale the $\mathrm{H} \beta$ result to the $\mathrm{H} \alpha$ and $\mathrm{H} \gamma$ lines using recombination theory (Storey \& Hummer 1995) assuming $T_{\mathrm{e}}=10000 \mathrm{~K}$ and $N_{\mathrm{e}}=$ $10000 \mathrm{~cm}^{-3}$, Case B: $I(\mathrm{H} \alpha) / I(\mathrm{H} \beta)=2.85$ and $I(\mathrm{H} \beta) / I(\mathrm{H} \gamma)=$ 2.13. ${ }^{3}$ This results in predicted continuum corrections for $\mathrm{F} 656 \mathrm{~N}$ of $0.005926 I(\mathrm{H} \alpha)$ and for F437N of $0.04709 I(H \gamma)$. The continuum relative to 4364 is obtained by multiplying the ratio of STIS observed fluxes of $\mathrm{H} \gamma / 4364$ by the continuum/ $\mathrm{H} \gamma$ value of 0.04709 . The differential extinction from 4364 to $4342 \AA$ is negligible for 7009 .

The continuum correction for $\mathrm{F} 502 \mathrm{~N}$ relative to [O III] 5008 is obtained by multiplying the ratio of STIS observed fluxes of $\mathrm{H} \beta / 5008$ by the continuum/ $\mathrm{H} \beta$ value of 0.02294 . Because the 5008 line is the strongest of the set, the continuum correction is extremely small; hence, it is safe to neglect the very small differential extinction from 4863 to $5008 \AA$.

While the above discussion is appropriate for correcting for the continuum in the specific WFPC2 filters, if comparison is made between individual filters, then an extinction correction would need to be considered (see Section 4).

Our original observing plan had been to use the WFPC2/F547M data to help determine the continuum emission. This does not appear to be a reliable method as judged by comparing our STIS and WFPC2 data. For instance, let us consider area E1. The observed F547M flux is $1.128 \times 10^{-12} \mathrm{erg} \mathrm{cm}^{-2} \mathrm{~s}^{-1}$. Using the continuum predictions of the theoretical model mentioned earlier for the mean wavelength $5468 \AA$ of this filter, the continuum emission at $5468 \AA$ relative to the $\mathrm{H} \beta$ line emission is $6.25 \times 10^{-4} \AA^{-1}$. To obtain the total continuum contribution in this filter, we multiply by the RECTW $=638.58 \AA$ and then multiply by the observed STIS $\mathrm{H} \beta$ flux of $1.507 \times 10^{-12} \mathrm{erg} \mathrm{cm}^{-2} \mathrm{~s}^{-1}$ to arrive at $6.014 \times 10^{-13} \mathrm{erg}$ $\mathrm{cm}^{-2} \mathrm{~s}^{-1}$. This is only 53 per cent of the observed F547M flux. In the above assessment, we have neglected the small differential extinction. Presumably the other $\sim 47$ per cent is due to line emission. We made a reality check of this number by using the digital version of the total system throughput for this filter and the measured line fluxes in Hyung \& Aller (1995b). The full width at 0.01 of the peak transmission of the F547M filter extends from 5002-5997 A.

\footnotetext{
${ }^{2}$ Note that RECTW is not the 'effective width of the bandpass' tabulated in table 6.1 and also shown as dashed rectangles in the Passband Plots of Filter Throughput (appendix 1) of the WFPC2 Instrument Handbook (Biretta et al. 2001).

${ }^{3}$ For conditions applicable to NGC 7009, we find using an online program (see Storey \& Hummer 1995) that the $I(\mathrm{H} \alpha) / I(\mathrm{H} \beta)$ ratio used here will depart by less than 1.75 per cent over the range $7500 \leqslant T_{\mathrm{e}} \leqslant 12500 \mathrm{~K}$ and $10^{3} \leqslant N_{\mathrm{e}} \leqslant 10^{5} \mathrm{~cm}^{-3}$. The $I(\mathrm{H} \beta) / I(\mathrm{H} \gamma)$ ratio will vary by $\leqslant 1$ per cent over the range $7500 \leqslant T_{\mathrm{e}} \leqslant 15000 \mathrm{~K}$ and $10^{2} \leqslant N_{\mathrm{e}} \leqslant 10^{6} \mathrm{~cm}^{-3}$.
}

As above, we account for the actual Doppler velocity of the source. The result is that by far the bulk of the line emission in F547M is due to the 5008 line; the ratio of the total continuum flux (calculated as described) to the scaled, transmitted 5008 line flux is 2.43 . We also include the next most significant contributors: He I 5877, He II 5413 and [ $\mathrm{Cl}$ III] 5519,39 . The estimated contribution then of lines is $\sim 35$ per cent of the total F547M emission. If the many other weaker lines were included, this value would increase somewhat. The main point of this exercise is to show that there can indeed be serious 'leakage' of 5008 line emission into the F547M data for NGC 7009 and no doubt for other PNs.

In addition to accounting for the continuum in each WFPC2 filter and the $\mathrm{H} \gamma$ leakage in $\mathrm{F} 437 \mathrm{~N}$, it is also necessary to account for the contribution of [N II] 6585.23- and 6549.86- $\AA$ emission into F656N. This is done by measuring the fluxes of these lines, as well as that of $\mathrm{H} \alpha$, in the areas E1 and WW. The method follows from that used for the $\mathrm{H} \gamma$ leakage in F437N. The Doppler corrected wavelengths for the three lines are determined and the relative throughputs applied for the F656N filter. These are 0.03645 and 0.4552 for the 6585 and $6550-\AA$ lines, respectively, relative to $\mathrm{H} \alpha$. Note that although the 6585 flux will always be larger than that of 6550, the latter contributes more to the observed F656N flux.

Table 1 shows the details of the comparison with the STIS overlay for E1 and WW for each of the WFPC2 narrow-band filters. Column 2 has the ratios of the observed line flux with STIS to the corresponding narrow band filter flux with WFPC2. Column 3 shows the flux ratio 'Contm./emission' line, derived from recombination theory and the WFPC2 filter profile. Columns 4 and 5 provide the observed line flux ratio derived from STIS data as modified passing through the pertinent WFPC2 filter profile. Column 6 then shows the 'Total' (of the previous columns 2 up to 5) for the STIS flux (including the theoretical continuum) divided by the flux observed in the narrow-band filter. The results for both areas are in close agreement; thus we use the combined area E1 + WW, which we call 'SUM', for our actual calibration to obtain observed line fluxes from the WFPC2 data. In the case of F437N and F502N, the total of the STIS emission plus inferred continuum exceeds the WFPC2 flux by a factor of 1.089 and 1.043 respectively, while for $\mathrm{F} 487 \mathrm{~N}$ and F656N, the total STIS emission plus continuum is less than the WFPC2 flux by factors of 1.032 and 1.037 respectively. From the SUM entry line for F437N in Table 1, we see that the relative strengths of the components comprising F437N are roughly in the proportions $0.71: 0.19: 0.10$ for the 4364 line : continuum : $\mathrm{H} \gamma$ leakage.

We use the results for the SUM area to define our best relationships for determining the necessary emission line fluxes from the WFPC2 data. Because the F437N corrections for the continuum and $\mathrm{H} \gamma$ leakage are tied to a measurement of a Balmer line flux, we use $\mathrm{H} \beta$ from the $\mathrm{F} 487 \mathrm{~N}$ image to measure its flux. Thus we arrive at equations (1)-(4).

$$
\begin{aligned}
& F(\mathrm{H} \alpha)=0.9531 \mathrm{~F} 656 \mathrm{~N}, \\
& F(\mathrm{H} \beta)=0.9482 \mathrm{~F} 487 \mathrm{~N}, \\
& \mathrm{~F}(5008)=1.041 \mathrm{~F} 502 \mathrm{~N}, \\
& \mathrm{~F}(4364)=1.089 \mathrm{~F} 437 \mathrm{~N}-0.03218 \times 10^{-0.124 c(\mathrm{H} \beta)} \mathrm{F} 487 \mathrm{~N} .
\end{aligned}
$$

Equation (4) requires further description. Both the $\mathrm{H} \gamma$ leakage and continuum corrections relative to $\mathrm{H} \gamma$ are known for the specific STIS-calibrated areas above. However, when we deal with other parts of the nebula observed with WFPC2 but not by the STIS slit, 
Table 1. Flux ‘calibration' of WFPC2 Filters with STIS data

\begin{tabular}{|c|c|c|c|c|c|}
\hline \multicolumn{6}{|c|}{ F437N } \\
\hline Location & $4364 / \mathrm{F} 437 \mathrm{~N}$ & Contm./4364 & $4342 / 4364$ & & Total/F437N \\
\hline E1 & 0.7834 & 0.2610 & 0.1402 & & 1.098 \\
\hline WW & 0.7623 & 0.2728 & 0.1467 & & 1.082 \\
\hline SUM & 0.7714 & 0.2677 & 0.1438 & & 1.089 \\
\hline \multicolumn{6}{|c|}{ F487N } \\
\hline Location & $4863 / \mathrm{F} 487 \mathrm{~N}$ & Contm./4863 & & & Total/F487N \\
\hline E1 & 0.9431 & 0.02207 & & & 0.9639 \\
\hline WW & 0.9520 & 0.02207 & & & 0.9730 \\
\hline SUM & 0.9482 & 0.02207 & & & 0.9691 \\
\hline \multicolumn{6}{|c|}{ F502N } \\
\hline Location & $5008 / \mathrm{F} 502 \mathrm{~N}$ & Contm./5008 & & & Total/F502N \\
\hline E1 & 1.031 & 0.00201 & & & 1.033 \\
\hline WW & 1.048 & 0.001984 & & & 1.050 \\
\hline SUM & 1.041 & 0.001995 & & & 1.043 \\
\hline \multicolumn{6}{|c|}{ F656N } \\
\hline Location & $6565 / \mathrm{F} 656 \mathrm{~N}$ & Contm./6565 & $6550 / 6565$ & $6585 / 6565$ & Total/F656N \\
\hline E1 & 0.9484 & 0.005926 & 0.00407 & 0.00106 & 0.9589 \\
\hline WW & 0.9564 & 0.005926 & 0.005538 & 0.001361 & 0.9687 \\
\hline SUM & 0.9531 & 0.005926 & 0.004913 & 0.001231 & 0.9646 \\
\hline
\end{tabular}

there is no direct way of knowing the observed $\mathrm{H} \gamma$ flux. Therefore, we make these corrections in terms of the observed $\mathrm{H} \beta$ flux, which may be assessed via the F487N data. Again, we use the theoretical flux ratio $\mathrm{H} \beta / \mathrm{H} \gamma=2.13$ but must correct for differential extinction between the two lines. In the presence of extinction, the observed $\mathrm{H} \gamma$ line will be weaker relative to the observed $\mathrm{H} \beta$ line (than the theoretical ratio). Therefore, the contribution to $\mathrm{F} 437 \mathrm{~N}$ will be less and thus the correction term should be less. The extinction is measured by the parameter $c(\mathrm{H} \beta)$, which is the logarithmic extinction correction at $\mathrm{H} \beta$, discussed in the next section. The constant 0.03218 in equation (4) was determined by requiring perfect agreement amongst all the pertinent observed fluxes for area SUM.

\section{EXTINCTION AND REDDENING CORRECTION}

Before deriving the $T_{\mathrm{e}}$ distribution from either the STIS or WFPC2 data, we first correct for extinction. This is calculated by comparing the observed $F(\mathrm{H} \alpha) / F(\mathrm{H} \beta)$ ratio with the theoretical ratio $I(\mathrm{H} \alpha) / I(\mathrm{H} \beta)$. Again, we use a value of 2.85 assuming $T_{\mathrm{e}}=$ $10000 \mathrm{~K}$ and $N_{\mathrm{e}}=10000 \mathrm{~cm}^{-3}$, Case B (Storey \& Hummer 1995). The extinction correction is done in terms of $c(\mathrm{H} \beta)$, given by the relationship

$\log [F(\lambda) / F(\mathrm{H} \beta)]=\log [I(\lambda) / I(\mathrm{H} \beta)]-f(\lambda) c(\mathrm{H} \beta)$,

where $f(\lambda)$ is the extinction curve. For the 4364-, 4863-, 4960-, 5008- and 6565 - $\AA$ lines, the respective values for $f(\lambda)$ are $0.124,0$, $-0.023,-0.034$ and -0.323 (Seaton 1979). This leads to

$c(\mathrm{H} \beta)=3.096 \log [F(\mathrm{H} \alpha) / F(\mathrm{H} \beta)]-1.408$.

The correction for extinction/reddening from observed to intrinsic flux for the 4364 and 5008 lines is then given by,

$I(4364)=\mathrm{F}(4364) 10^{1.124 c(\mathrm{H} \beta)} ; \quad I(5008)=\mathrm{F}(5008) 10^{0.966 c(\mathrm{H} \beta)}$.

Note that the first of these was used to derive equation (4).

For the STIS data, we binned the pixels along the slit into tiles that are 0.5 arcsec square (matching the slit width). The CS and
STIS fiducial bars are excluded. This produced $c(\mathrm{H} \beta)$ results for 45 tiles, which are the same set used later for the $T_{\mathrm{e}}$ analysis. The statistics without regard to any weighting for brightness are: the average $c(\mathrm{H} \beta)$ is 0.1032 ; the median is 0.1038 ; and the values range from 0.0239 to 0.1592 . The median and average are small and close to previous spectroscopic values, e.g. 0.12 (Barker 1983, and references therein).

For the WFPC2 data, we work with the individual pixels. There were a few negative $c(\mathrm{H} \beta)$ values, which are replaced with a value of zero. There also were spuriously high $c(\mathrm{H} \beta)$ values inferred in the fast, low-ionization emission regions (FLIERS), particularly on the west side. The reason for this is that the [N II] 6550-, 6585- $\AA$ lines are very much stronger in the FLIERS. This can be seen from the paper by Balick et al. (1998) in their colour image (fig. 1) and emission-line profiles (fig. 3). The leakage by the 6550-, 6585- $\AA$ lines into F656N enormously exceeds the 'calibration' shown in Table 1. The application of equation (1) overestimates $F(\mathrm{H} \alpha)$ in the FLIERS, resulting in a spuriously high $F(\mathrm{H} \alpha) / F(\mathrm{H} \beta)$ and thus $c(\mathrm{H} \beta)$, reaching as high as 0.456 . With the guidance of the STIS slit overlay, we chose to cap the $c(\mathrm{H} \beta)$ value in the vicinity of the FLIERS at a value of 0.14 . This value is not crucial and could have been set between $0-0.2$ because extinction is low for NGC 7009. As will be seen in the next section, it matters little for the subsequent temperature analysis.

\section{ELECTRON TEMPERATURE DETERMINATION}

The electron temperature $T_{\mathrm{e}}$ is derived from the intrinsic ratio $I(5008) / I(4364)$ using the following relation,

$T_{\mathrm{e}}=32966 /\{\ln [I(5008) / I(4364)]-1.701\}$.

Effective collision strengths are from Burke, Lennon \& Seaton (1989) for $T_{\mathrm{e}}=10^{4} \mathrm{~K}$. Transition probabilities ( $A$-values) are from Froese Fischer \& Saha (1985). Note that this holds in the low- $N_{\mathrm{e}}$ limit, which should be valid for NGC 7009 where $N_{\mathrm{e}}$ values are less than the critical densities $\left(N_{\text {crit }}\right)$ for these lines. The lowest $N_{\text {crit }} \sim 6.4 \times 10^{5} \mathrm{~cm}^{-3}$ for the 5008 line, which is well above $N_{\mathrm{e}}$ values determined (e.g. Hyung \& Aller 1995a,b). 


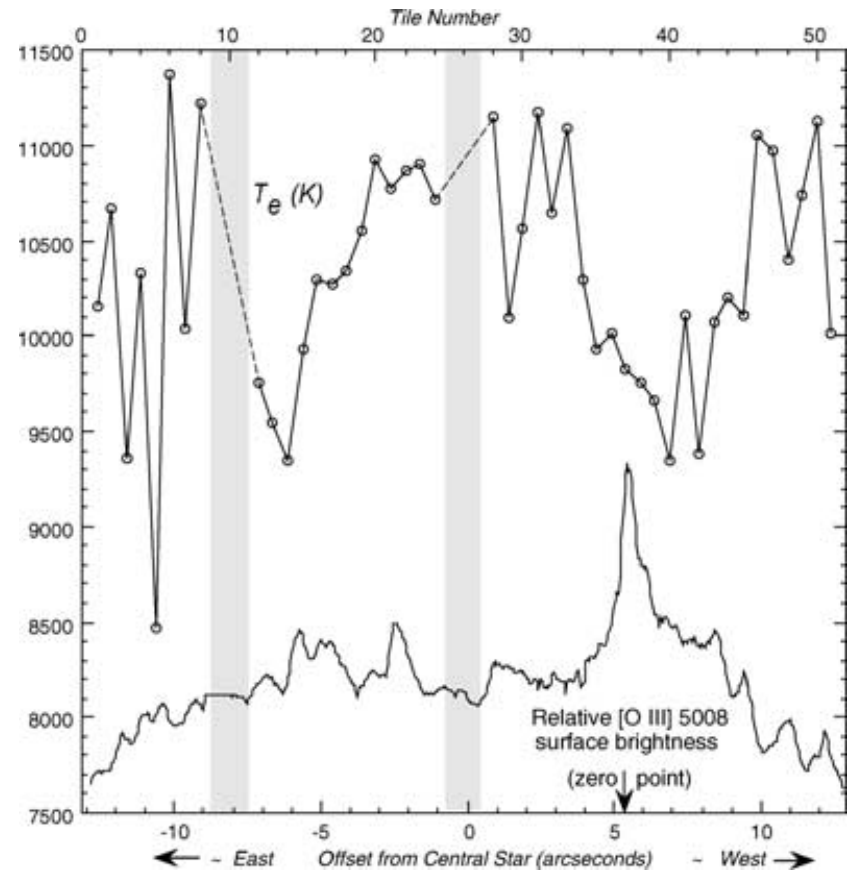

Figure 1. Plot of $T_{\mathrm{e}}$ determined from the [O III] 4364/5008 flux ratio versus position along the STIS long-slit. The analysis is in terms of tiles that are 0.5 arcsec square (matching the slit width). There are 45 tiles with reliable measurements that exclude the grey areas. The open circles represent the individual tiles plotted at their midpoint. The dashed straight lines are interpolations across the central star tiles (\#25-27) and across the east fiducial bar tiles $(9-11)$. Tile \# increases from $\sim$ east to $\sim$ west. The bottom curve shows the observed relative [O III] 5008- $\AA$ flux displayed unsmoothed at the pixel level.

For the STIS data, we continue with the analysis using the 45 tiles along the slit described above. Equations (6)-(8) are applied to the four emission lines to derive $T_{\mathrm{e}}$. Fig. 1 shows the distribution of $T_{\mathrm{e}}$ versus position along the slit. This is shown relative to the closest approach of the CS to our slit (recall the CS was placed off the slit edge). The open circles represent the individual tiles plotted at their midpoint. The dashed lines are a linear interpolation across the tiles that were deemed to have unreliable measurements because of proximity to the CS (tile \#25-27) and the east fiducial bar (9-11). There appears to be some symmetry with respect to the CS. From a more or less flat $T_{\mathrm{e}} \sim 10800 \mathrm{~K}$ over the centre tiles, there is a decrease to $\sim 9500 \mathrm{~K}$ and then a rise again to roughly $10800 \mathrm{~K}$ in both the east and west with increasing distance from the star. The situation is less clear on the east side, partly because of the lack of data at the east fiducial bar. In the next section, we will evaluate this distribution in terms of $T_{\mathrm{e}}$ variations.

A plot of the observed relative [O III] $5008 \AA$ flux is also shown in Fig. 1. This curve is displayed (unsmoothed) at the pixel level providing 0.05 arcsec spatial resolution along the slit. There are some noteworthy oscillations between $\sim 8-13$ arcsec west of the CS. There appears to be 4 cycles with a period between $\sim 1-1.5 \operatorname{arcsec}$ per cycle. These reach a factor of $\sim 2$ brightness contrast between adjacent local maxima and minima. The structure is very repeatable when compared to the corresponding run of observed $\mathrm{H} \alpha$ and $\mathrm{H} \beta$ flux. Because these oscillations do not appear to be correlated with the $T_{\mathrm{e}}$ distribution and are not present in the flux ratios of 5008 to the Balmer lines, they must be due to a column density (or $\int N_{\mathrm{e}}^{2} \mathrm{~d} l$ ) modulation. Such a pattern might result from episodic mass loss.
We find very similar spatial behavior, including this tantalizing oscillatory pattern, from our WFPC2 F502N, F487N and F656N data when we overlay the STIS slit. We note that this result from our 1D cut is insufficient to establish a case for episodic mass loss.

Using the WFPC2 data, the path to $T_{\mathrm{e}}$ is more uncertain than is the case with our STIS measurements. However, the WFPC2 data cover a much larger area of the nebula. Equations (1)-(4) and (6)-(8) are used to derive $T_{\mathrm{e}}$. In Fig. 2, we present the $T_{\mathrm{e}}$ map at a resolution of 0.1 arcsec. The outer region with white and black speckles is omitted from the analysis. The $\mathrm{S} / \mathrm{N}$ in $\mathrm{F} 437 \mathrm{~N}$ is generally too low out there to obtain reliable $T_{\mathrm{e}}$ information. Nevertheless, with a hint of the ansae, this area helps visually identify the major axis. We note that by binning the data in the outlying areas, the $\mathrm{S} / \mathrm{N}$ may be improved. For the purposes of this paper, we restrict the analyses to the interior main body of the nebula with the highest $\mathrm{S} / \mathrm{N}$, where there is sufficient area to address temperature fluctuations in this nebula.

The high $T_{\mathrm{e}}$ white region immediately surrounding the CS is the result of diffracted or scattered stellar continuum. Equation (4), which corrects for the continuum in the F437N filter, does not account for stellar continuum emission. The result is a spuriously high $F$ (4364) leading to the spuriously high values for $T_{\mathrm{e}}$. Further out, the temperatures show the red and yellow colour code. The pattern that looks like an 'H'-shape region, or for the imaginative, the 'Batman symbol' appears to be point symmetric with respect to the CS. This region is likely to be at the elevated $T_{\mathrm{e}} \mathrm{S}$ indicated because of the agreement in this central region with the STIS results shown in Fig. 1. The full STIS long slit is shown superimposed on Fig. 2. The tiles along the slit are entirely within the inner higher $\mathrm{S} / \mathrm{N}$ region. The distances from the CS to the centres of the two symmetrical blue 'lakes' that the STIS slit passes through are roughly 6.6 arcsec. That distance corresponds to $\sim 13$ tiles on Fig. 1, where the CS location is within tile 26. Thus the troughs in the $T_{\mathrm{e}}$ structure in Fig. 1 near tile 13 and 39 correspond with the blue lakes. The temperatures also start to rise again further out as shown by the green colour. However, there is not a prominent increase in $T_{\mathrm{e}}$ with increasing distance from the CS to the levels indicated from the $T_{\mathrm{e}}$ distribution along the slit in Fig. 1.

The determination of $T_{\mathrm{e}}$ from $I(5008) / I(4364)$ is not sensitive to the extinction correction for NGC 7009 because extinction is small. We may demonstrate this with an example using a $c(\mathrm{H} \beta)$ of 0.1 , which is close to the average value. Let us also use a case that results in a representative temperature $10000 \mathrm{~K}$. The observed $F(5008) /$ $F(4364)$ would be 153.543 to result in $I(5008) / I(4364)=148.057$, which results in $T_{\mathrm{e}}=10000 \mathrm{~K}$ by equation (8). If $c(\mathrm{H} \beta)$ were respectively 0.0 or 0.2 instead with the same observed flux ratio, the resulting $T_{\mathrm{e}}$ would be 9891 and $10112 \mathrm{~K}$. Thus were we to carry out this analysis with a constant $c(\mathrm{H} \beta)=0.1$, or no extinction correction at all, the results will be hardly altered.

\section{FRACTIONAL MEAN-SQUARE TEMPERATURE VARIATIONS}

Our STIS and WFPC2 analysis above present results in the plane of the sky. The observations here do not address temperature fluctuation along the line of sight, which may be characterized in terms of the average temperature $T_{0}$ and fractional mean-square $T_{\mathrm{e}}$ variation $\left(t^{2}\right)$ as defined by Peimbert (1967).

$T_{0}=\frac{\int T_{\mathrm{e}} N_{\mathrm{e}} N_{\mathrm{i}} \mathrm{d} V}{\int N_{\mathrm{e}} N_{\mathrm{i}} \mathrm{d} V}$, 
$t^{2}=\frac{\int\left(T_{\mathrm{e}}-T_{0}\right)^{2} N_{\mathrm{e}} N_{\mathrm{i}} \mathrm{d} V}{T_{0}^{2} \int N_{\mathrm{e}} N_{\mathrm{i}} \mathrm{d} V}$,

where $N_{\mathrm{i}}$ is the ion density $\mathrm{N}\left(\mathrm{O}^{++}\right)$. The integration in equations (9) and (10) is over the column defined by each pixel (or tile for the STIS analysis), and along the line of sight (los). We are unable to measure the $t^{2}$ along the los for any column (cross-section of 1 pixel or tile). If there are $t^{2}$ along the los, we can say that $T(4364 / 5008)>T_{0}$ (e.g. Peimbert 1967; Rubin et al. 1998).

For each pixel/tile, we have calculated $T(4364 / 5008)$. Then the intrinsic flux $I$ (5008), fully correcting $F(5008)$ for extinction (see equation 7), in each pixel/tile is used in conjunction with $T_{\mathrm{e}}=$ $T(4364 / 5008)$ for that pixel/tile, and assumed constant along the los, to derive the following:

$$
\begin{aligned}
I(5008) & =K(5008) \int N_{\mathrm{e}} N_{\mathrm{i}} T_{\mathrm{e}}^{-0.5} \exp \left(-\chi / k T_{\mathrm{e}}\right) \mathrm{d} l \\
& =K\left(5008, T_{\mathrm{e}}\right) \int N_{\mathrm{e}} N_{\mathrm{i}} \mathrm{d} l .
\end{aligned}
$$

Here $\chi$ is the excitation energy above the ground state for the upper level of the 5008 transition, $k$ is Boltzmann's constant, $K(5008)$ is known from atomic data, and $K\left(5008, T_{\mathrm{e}}\right)$ has finally incorporated the known $T_{\mathrm{e}}$ factor with the atomic constants. Here we again make the safe assumption of the low- $N_{\mathrm{e}}$ limit (negligible collisional deexcitation) discussed earlier.

Let us now define analogous terms to represent the average $T_{\mathrm{e}}$ $\left(T_{0, A}\right)$ and fractional mean-square $T_{\mathrm{e}}$ variation $\left(t_{A}^{2}\right)$ in the plane of the sky.

$T_{0, A}=\frac{\iint T_{\mathrm{e}} N_{\mathrm{e}} N_{\mathrm{i}} \mathrm{d} l \mathrm{~d} A}{\iint N_{\mathrm{e}} N_{\mathrm{i}} \mathrm{d} l \mathrm{~d} A}$,

$t_{A}^{2}=\frac{\iint\left(T_{\mathrm{e}}-T_{0, A}\right)^{2} N_{\mathrm{e}} N_{\mathrm{i}} \mathrm{d} l \mathrm{~d} A}{T_{0, A}^{2} \iint N_{\mathrm{e}} N_{\mathrm{i}} \mathrm{d} l \mathrm{~d} A}=\frac{\iint T_{\mathrm{e}}^{2} N_{\mathrm{e}} N_{\mathrm{i}} \mathrm{d} l \mathrm{~d} A}{T_{0, A}^{2} \iint N_{\mathrm{e}} N_{\mathrm{i}} \mathrm{d} l \mathrm{~d} A}-1$,

where $\mathrm{d} A$ represents an element of surface area in the plane of the sky and the integration over $\mathrm{d} l$ is for each pixel/tile along the los.

\subsection{WFPC2 analysis}

For this part, the image in Fig. 2 was further rotated so that the NGC 7009 major axis was aligned with the $x$-axis. We obtained from this an array of intrinsic fluxes $I$ (4364) and $I(5008)$ for input to our own analysis code. For each of the above set of pixel-by-pixel solutions, we have the $[\mathrm{O} \mathrm{III}] T_{\mathrm{e}}$ (that assumed no $T_{\mathrm{e}}$ variations along the los). A circular region (radius of 10 pixels $=1$ arcsec) surrounding the $\mathrm{CS}$ is excluded from further calculations. Additionally, we exclude pixels near the periphery, where the $\mathrm{S} / \mathrm{N}$ for the $4364-\AA$ line is poor.

Calculations for $T_{0, A}$ and $t_{A}^{2}$ were performed using various distances from the CS $\left(R_{\text {outer }}\right)$. This was performed in several ways: assuming concentric circular shapes, and assuming concentric elliptical shapes, which is perhaps more realistic for this source. The bottom-line results are virtually unchanged between these different methods. In Figs 3 and 4, we present our results for $T_{0, A}$ and $t_{A}^{2}$ for an elliptical shape with an axial ratio of minor/major $=0.808$, matching our $T_{\mathrm{e}}$ map (Fig. 2). The abscissa $R_{\text {outer }}=\sqrt{a_{\text {outer }} b_{\text {outer }}}$, where $a_{\text {outer }}$ and $b_{\text {outer }}$ are the semi-major and semi-minor axes. Out to the most distant elliptical contour used in our analysis ( $a_{\text {outer }}=$ $\left.14.5 \mathrm{arcsec}, b_{\text {outer }}=11.7 \operatorname{arcsec}, R_{\text {outer }}=13.0 \operatorname{arcsec}\right)$, there are 52995 good pixels with all [O III] $T_{\mathrm{e}}$ values between $5531-16382 \mathrm{~K}$.
The dashed lines pertain to values for $T_{0, A}$ and $t_{A}^{2}$ for the entire area inside the ellipse with $a_{\text {outer }}, b_{\text {outer }}$, and is plotted at the single distance $R_{\text {outer }}$ (again excluding the CS) and represent cumulative results. The solid lines pertain to $T_{0, A}$ and $t_{A}^{2}$ in the annular area between two adjacent ellipses separated by 5 pixels $(0.5$ arcsec $)$ along the major axis. This is plotted as a single point at a distance $R_{\text {centroid }}=\sqrt{0.5\left(a_{\text {outer }} b_{\text {outer }}+a_{\text {inner }} b_{\text {inner }}\right)}$.

There is nearly a monotonic decline in $T_{0, A}$ with increasing distance (as characterized for the ellipses) from the CS. The results indicate very small values $-<0.012-$ for $t_{A}^{2}$ throughout. The overall $T_{0, A}=9912 \mathrm{~K}, t_{A}^{2}=0.00360$ for the $52995 \operatorname{good}$ pixels within the most distant elliptical contour used (excluding CS region). There is a steep climb in $t_{A}^{2}$ in the outer elliptical annuli (solid line in Fig. 4), although this may be due to noisier data at larger distances from the CS.

\subsection{STIS analysis}

Our STIS data allow an even more direct determination of $T_{\mathrm{e}}$ and hence of $T_{0, A}, t_{A}^{2}$, albeit for a much smaller area than with WFPC2. The average [O III] temperature $T_{0, A}$ using 45 tiles (excluding the $\mathrm{CS}$ and fiducial bar) is $10139 \mathrm{~K} ; t_{A}^{2}$ is 0.00350 . The median value (without regard to proper weighting) is $10295 \mathrm{~K}$. All four emission lines peak in tile 37 , with tile 38 a close runner-up. This is where our slit crosses the 'ridge' near the blue-green (He II 4687-[O III] 5008) boundary in the three-colour image (Balick et al. 1998) at roughly 5-6 arcsec from the CS. These tiles have relatively low [O III] $T_{\mathrm{e}}$ values of 9823 and $9755 \mathrm{~K}$, respectively (see Fig. 1). No doubt, the enhancement in line emission here is due to a greater column density (and/or higher density) and not temperature. As determined from the extinction-corrected 5008 surface brightness (equation 11), these tiles have the highest $\left(\int N_{\mathrm{e}} N_{\mathrm{i}} \mathrm{d} l\right)$-weighting in equations (12) and (13).

We note that the single pixel (out of 52 995) with the highest 5008 surface brightness also has the highest $\left(\int N_{\mathrm{e}} N_{\mathrm{i}} \mathrm{d} l\right)-,\left(\int T_{\mathrm{e}} N_{\mathrm{e}} N_{\mathrm{i}} \mathrm{d} l\right)-$ and $\left(\int T_{\mathrm{e}}^{2} N_{\mathrm{e}} N_{\mathrm{i}} \mathrm{d} l\right)$-weighting in the WFPC2 analysis. This pixel is located with respect to the CS 5.9 arcsec along the west major axis and 4.7 arcsec orthogonally below and is associated with the 'ridge' near the blue-green boundary of the Balick et al. (1998) picture.

\section{DISCUSSION AND CONCLUSIONS}

We determined the electron temperature from the flux ratio of [O III] (4364/5008) using both STIS and WFPC2 observations of NGC 7009. The resulting $T_{\mathrm{e}}$ values from both the WFPC2 map and the distribution along the STIS slit do not vary much, with almost all values between $9000-11000 \mathrm{~K}$. The higher $T_{\mathrm{e}}$ values are closer to the central star and appear, from WFPC2 images, to coincide with the inner $\mathrm{He}^{++}$-zone. The temperature is higher in the $\mathrm{He}^{++}$-zone because heating is stronger due to radiation beyond $54.4 \mathrm{eV}$, while the cooling is less efficient as $\mathrm{O}^{++}$gives way to $\mathrm{O}^{+3}$. Models show that some $\mathrm{O}^{++}$remains inside the $\mathrm{He}^{++}$zone, so the higher temperature there will be reflected in T(4364/5008) - see, for example, figs 11 and 14 of Harrington et al. (1982).

The observations here do not address $T_{\mathrm{e}}$ fluctuation along the line of sight. We assume for each square column (projection of 1 pixel for the WFPC2 data and 1 tile for the STIS data on the planeof-the-sky) that the plasma along the line of sight is isothermal at the $\mathrm{T}(4364 / 5008)$. The analysis of both data sets for the average $T_{\mathrm{e}}$ and fractional mean-square $T_{\mathrm{e}}$ variations in the plane-of-the-sky, which we call $T_{0, A}$ and $t_{A}^{2}$, gave consistent results. For the WFPC2 


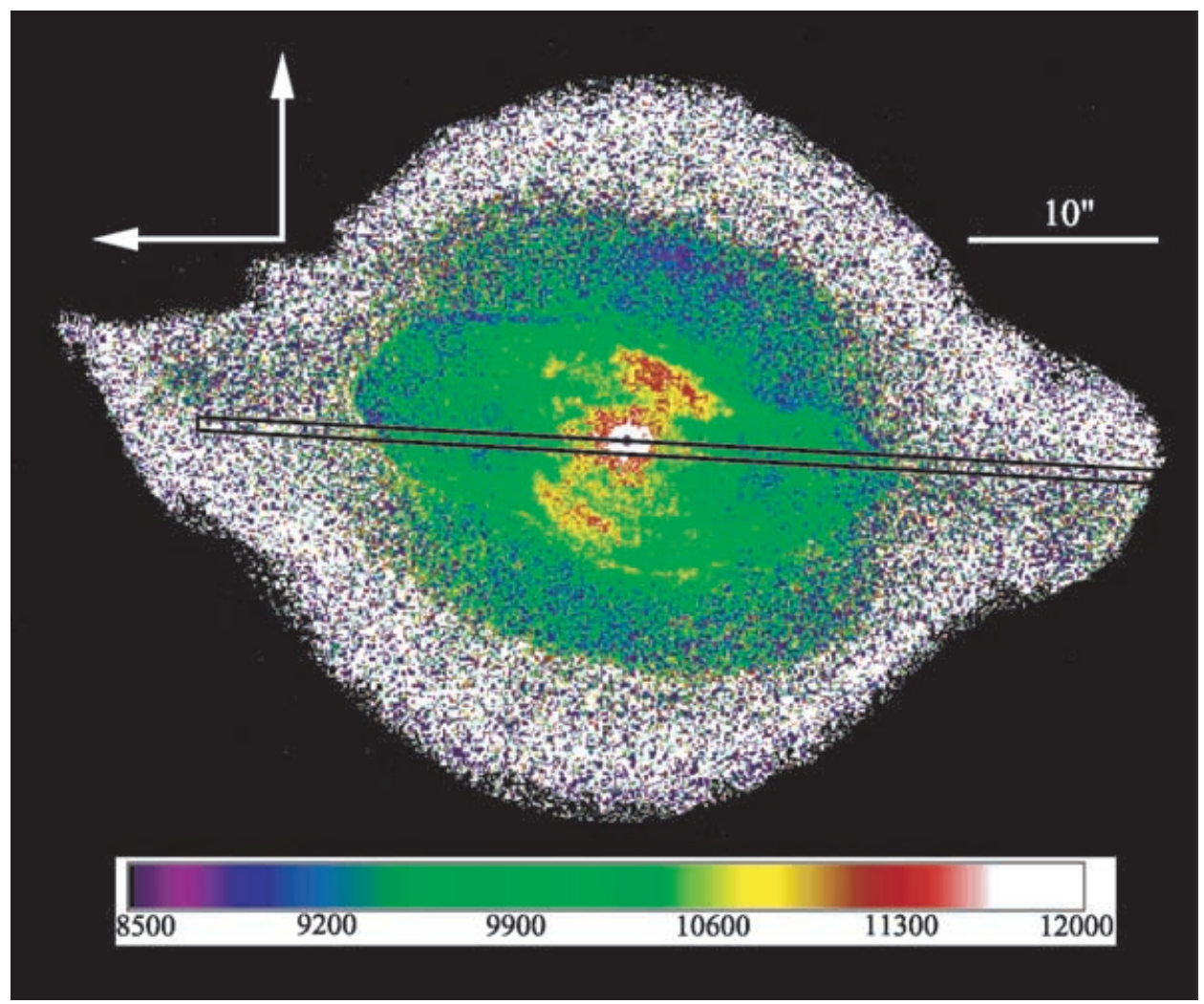

Figure 2. This is a pixel-by-pixel map of the electron temperature $T_{\mathrm{e}}$ (colour-bar scale) as determined from the ratio of extinction corrected fluxes of [O III] $(4364 \AA) /(5008 \AA)$. The STIS long-slit (52 arcsec $\times 0.5 \mathrm{arcsec})$ is aligned and superimposed on the image. The area representing the 0.5 -arcsec-wide slit is shown fully unobstructed. The circumscribed black boundary lines are not part of the STIS slit. North is up and east to the left.

analysis, the preferred method used concentric elliptical contours to conform to the natural elliptical shape of the nebula. For $T_{0, A}$, the STIS average was $10139 \mathrm{~K}$, while the WFPC2 overall average to the outermost elliptical contour considered was $9912 \mathrm{~K}$. Within the contours closer to the central star, $T_{0, A}$ is higher, as depicted in Fig. 3 . In all the statistical analyses, a region around the CS is excluded. For both STIS and WFPC2, $t_{A}^{2}$ is found to be very small, $<0.012$ everywhere. Using STIS, we obtained 0.0035 , which is close to the WFPC2 overall value of 0.0036 within the most distant elliptical contour used.

Because the continuum correction for the $\mathrm{F} 437 \mathrm{~N}$ data is a major source of uncertainty in the process of deriving $T_{\mathrm{e}}$ from the WFPC2 observations, we attempted to assess this using another approach. Instead of estimating the continuum correction theoretically for F437N, we assume that STIS and WFPC2 are perfectly calibrated. By this we mean that the total STIS emission/F437N in Table 1 should be unity (instead of 1.089). We then assume that the continuum emission contribution to the common WFPC2/STIS area SUM (see Section 3 and Table 1) be the remaining amount after accounting for the STIS 4364 and $\mathrm{H} \gamma$ emission as they would be observed passing through the F437N/telescope system. This reduces the Contm./4364 value for SUM in Table 1 to 0.1525 and alters the proportions that would comprise the F437N emission to be $0.77: 0.12: 0.11$ for the 4364 line : continuum : H $\gamma$ leakage. This treatment results in a modification of equation (4) with the factor 1.089 becoming 1.0 and the factor 0.03218 becoming 0.02316

We carried out the subsequent analysis with just this change to equation (4). The results were that the highest $T_{\mathrm{e}}$ values were low- ered several hundred degrees while the lowest $T_{\mathrm{e}}$ values were raised several hundred degrees. Consequently, the effect was to decrease $t_{A}^{2}$ values somewhat. Repeating the elliptical annuli analysis, we found the WFPC2 overall value of 0.00303 (decreased from 0.00360) within the most distant elliptical contour used. For the individual annuli, $t_{A}^{2}<0.01$ everywhere. For $T_{0, A}$, the WFPC2 overall average to the outermost elliptical contour considered increased slightly to $9940 \mathrm{~K}$ (from $9912 \mathrm{~K}$ ). Essentially, there were only minor changes to the curves depicted in Figs 3 and 4. We are led from the above computations to conclude that our results are robust and not sensitive in the situation considered here to the correction for continuum emission in the $\mathrm{F} 437 \mathrm{~N}$ data.

Fluctuations in $T_{\mathrm{e}}$ along the los are inevitable. We can make some comments about how our results for $t_{A}^{2}$ might be adjusted by $T_{\mathrm{e}}$ variations along the los. The relationship between $T(4364 / 5008)$ and $T_{0}$ is

$$
T(4364 / 5008)=T_{0}\left[1+0.5\left(91200 / T_{0}-3\right) t^{2}\right],
$$

(e.g. Peimbert 1967; Rubin 1969). For a given column, let us take a representative $T(4364 / 5008)$ of $10100 \mathrm{~K}$, which is close to the average $T_{0, A}$ for the STIS analysis. If $T_{0}$ were $9000 \mathrm{~K}$, then $t^{2}=$ 0.0343 along that los in the column; if $T_{0}$ were $8000 \mathrm{~K}$, then $t^{2}=$ 0.0625 . If we assume that generally a given los column will be characterized by $T_{0}=9000 \mathrm{~K}, 1100 \mathrm{~K}$ lower than $T(4364 / 5008)$, then according to the left-hand side form of equation (13), one would expect $t_{A}^{2}$ to be a factor of 1.26 larger. This is due to using the lower 9000-K temperature $\left(T_{0}\right)$ in the denominator instead of the $10100-\mathrm{K}$ temperature $T(4364 / 5008)$. Our conclusion will still remain that $t_{A}^{2}$ is very small as measured by the $H S T$ data here. 


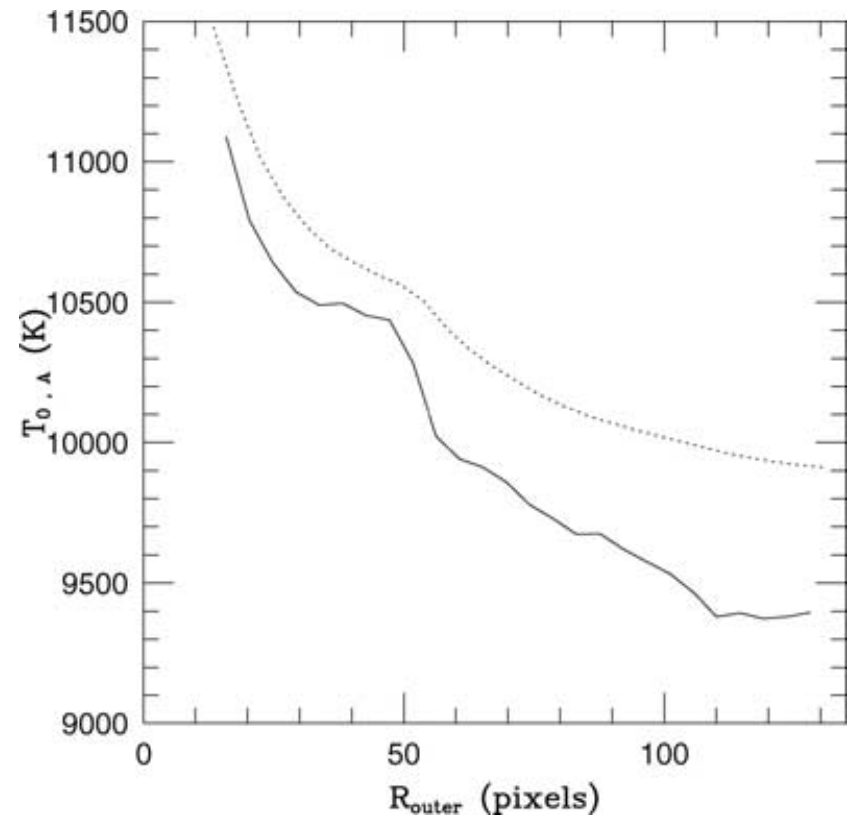

Figure 3. $T_{0, A}$ versus $R_{\text {outer }}=\sqrt{a_{\text {outer }} b_{\text {outer }}} . R_{\text {outer }}$ is measured from the central star in pixels with 10 pixels equal 1 arcsec. The dashed curve pertains to values for $T_{0, A}$ for the entire area interior to the ellipse with $a_{\text {outer }}, b_{\text {outer }}$ and is shown as a point at $R_{\text {outer }}$. The solid line pertains to $T_{0, A}$ in the annular area between two adjacent ellipses. This point is plotted at the annulus area centroid (see text). The step along the major axis between two successive elliptical annuli is five pixels; along the minor axis, the step is 4.04 pixels.

We do not have the data here to characterize $T_{\mathrm{e}}$ variations in $3 \mathrm{D}$. One way that this may be realized is to consider the following case. If every single pixel/tile los-column were to have $T(4364 / 5008)=$ $10100 \mathrm{~K}, T_{0}=9000 \mathrm{~K}$ and $t^{2}=0.0343$, the analysis for variations in the plane-of-the-sky that we present here will result in $T_{0, A}=$ $10100 \mathrm{~K}$ and $t_{A}^{2}=0$. However, the overall true $3 \mathrm{D} T_{0}=9000 \mathrm{~K}$ and $t^{2}=0.0343$. It is useful to define an overall $3 \mathrm{D}$ average $T_{\mathrm{e}}\left(T_{0, V}\right)$ and fractional mean-square $T_{\mathrm{e}}$ variation $\left(t_{V}^{2}\right)$. These single values apply

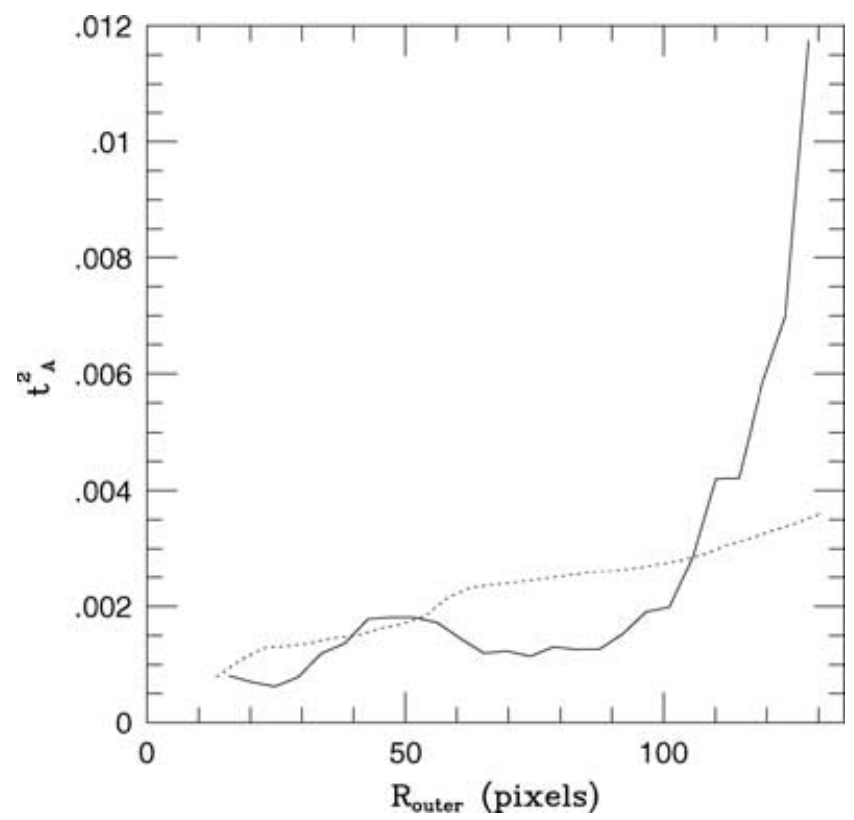

Figure 4. Same as Fig. 3 except for $t_{A}^{2}$ instead of $T_{0, A}$. for the entire source. Equations (9) and (10) define these specific values when the integration is over the entire volume. For the simple, contrived case above, $T_{0, V}=9000 \mathrm{~K}$ and $t_{V}^{2}=0.0343$. We note that for a spatially unresolved object (total integrated fluxes observed in the aperture), $t_{V}^{2}=t^{2}$ and a calculation of $t_{A}^{2}$ is meaningless.

Our measurements of $T_{\mathrm{e}}$ reported here are an average along each line of sight. Because each element of area treated in the plane of the sky represents a column which has already created a spatially averaged temperature along the los (e.g. see fig. 1 in Rubin 1969), it is likely that the value for $t_{V}^{2}$ is substantially higher than $t_{A}^{2}$. Measurements of $t^{2}$ along various sightlines appear to be the most direct way to reliably gauge $t_{V}^{2}$. Therefore, despite finding remarkably low $t_{A}^{2}$, we cannot completely rule out temperature fluctuations along the los as the cause of the large abundance discrepancy between heavy element abundances inferred from collisionally excited emission lines compared to those derived from recombination lines. Further work, beyond the scope of this paper, is underway that will use modelling as well as additional observational data in an effort to better determine the relationship between $t^{2}, t_{A}^{2}$ and $t_{V}^{2}$.

Judging from both the shape of the inner parts of the nebula and echelle observations (Balick, Preston \& Icke 1987) of NGC 7009, the inner regions of the nebula are well modelled as the projected surface of a prolate ellipsoid. If this is true, then most of the lines of sight of interest in this paper intersect two thin sheets of emitting gas. Thus the temperature fluctuations along the los, if they exist, lie within these sheets. The internal physical structure within each of these sheets is probably not resolvable either in extant images or through high-dispersion spectroscopic observations.

Hydrodynamic models suggest that this ellipsoidal sheet consists of nebular material compressed by the supersonic growth of the hot (optically invisible) wind-heated bubble inside the surfaces. Models predict that this compressed gas cools efficiently and, to the first order, is isothermal except, perhaps, in a weak shock at the leading (outer) edge. Dopita (1997) has argued that the emission lines from such shocks are all but indiscernible since the integrated energy density of the shock is small compared to other energies that can excite the emission lines. However, the trailing edge of the compressed sheet is generally assumed to be a simple contact discontinuity ('CD') across which no pressure gradient exists. In these models, in which magnetic fields and thermal conduction are not considered, no $T_{\mathrm{e}}$ gradient is expected across the $\mathrm{CD}$. Recent x-ray observations (Chu et al. 2001; Kastner et al. 2000; Kastner, Vrtilek \& Soker 2001) call this description of the CD into question. In particular, the edges of the hot bubble in contact with the $\mathrm{CD}$ are much cooler and denser than expected. It seems possible that heat from the hot bubble is being conducted from the bubble into the trailing edge of the sheet. Hence a steep temperature gradient may exist over a thin region from the bubble $\left(T_{\mathrm{e}} \sim 10^{6} \mathrm{~K}\right)$ into the compressed gas $\left(T_{\mathrm{e}} \sim 10^{4} \mathrm{~K}\right)$.

\section{ACKNOWLEDGMENTS}

This paper is based on observations made with the NASA/ESA Hubble Space Telescope, obtained at the Space Telescope Science Institute (ST ScI). Support for proposal \#GO-8114 was provided through a grant from ST ScI. ST ScI is operated by the Association of Universities for Research in Astronomy, Inc., under NASA contract NAS5-26555. We are grateful to Aaron Svoboda for creating the $\mathrm{C}^{++}$code PIXHUNTER and to the NASA Undergraduate Research Program (NASA-USRP), which made his 2001 summer work at NASA/Ames possible. We thank the referee for useful comments. Valuable contributions were made 
during early stages by Chris Ortiz and John Nguyen. RHR acknowledges support from the Long-Term Space Astrophysics (LTSA) program, NASA/Ames Research Centre contract NCC29018 with Orion Enterprises, and thanks Scott McNealy for providing a Sun workstation.

\section{REFERENCES}

Balick B., Preston H. L., Icke V., 1987, AJ, 94, 1641

Balick B., Alexander J., Hajian A. R., Terzian Y., Perinotto M., Patriarchi P., 1998, AJ, 116, 360

Barker T., 1983, ApJ, 267, 630

Biretta J. et al. 2001, WFPC2 Instrument Handbook, Version 6.0. STScI, Baltimore, p. 317

Burke V. M., Lennon D. J., Seaton M. J., 1989, MNRAS, 236, 353

Chu Y.-H., Guerrero M. A., Gruendl R. A., Williams R. M., Kaler J. B., 2001, ApJ, 553, L69

Dopita M. A., 1997, ApJ, 485, L41

Froese Fischer C., Saha H. P., 1985, Phys. Scr., 32, 181

Grevesse N., Sauval A. J., 1998, Space Sci. Rev., 85, 161

Harrington J. P., Seaton M. J., Adams S., Lutz J. H., 1982, MNRAS, 199, 517

Henry R. B. C., Worthey G., 1999, PASP, 111, 919

Hyung S., Aller L. H., 1995a, MNRAS, 273, 958

Hyung S., Aller L. H., 1995b, MNRAS, 273, 973

Kastner J. H., Soker N., Vrtilek S. D., Dgani R., 2000, ApJ, 545, L57

Kastner J. H., Vrtilek S. D., Soker N., 2001, ApJ, 550, L189

Kingdon J. B., Ferland G. J., 1998, ApJ, 506, 323

Kingsburgh R. L., Barlow M. J., 1994, MNRAS, 271, 257

Kwitter K. B., Henry R. B. C., 1998, ApJ, 493, 247

Leitherer C. et al., 2001, STIS Instrument Handbook, Version 5.1. STScI, Baltimore

Liu X.-W., 2002, in ASP Conf. Ser. Planetary Nebulae, Their Evolution and Role in the Universe. Astron. Soc. Pac., San Francisco, submitted

Liu X.-W., Storey P. J., Barlow M. J., Clegg R. E. S., 1995, MNRAS, 272, 369

Liu X.-W., Storey P. J., Barlow M. J., Danziger I. J., Cohen M., Bryce M., 2000, MNRAS, 312, 585

Liu X.-W., Luo S.-G., Barlow M. J., Danziger I. J., Storey P. J., 2001, MNRAS, 327, 141

Luo S.-G., Liu X.-W., Barlow M. J., 2001, MNRAS, 326, 1049

Peimbert M., 1967, ApJ, 150, 825

Péquignot D. et al., 2002, Rev. Mex. Astron. Astrofis. Ser. Conf., 12, 142

Petersen C. C., Brandt J. C., 1998, Hubble Vision. Further Adventures with the Hubble Space Telescope, 2nd edn, Cambridge Univ. Press

Rubin R. H., 1969, ApJ, 155, 841

Rubin R. H., Martin P. G., Dufour R. J., Ferland G. J., Baldwin J. A., Hester J. J., Walter D. K., 1998, ApJ, 495, 891

Schneider S. E., Terzian Y., Purgathofer A., Perinotto M., 1983, ApJS, 52, 399

Seaton M. J., 1979, MNRAS, 187, 73P
Shaver P. A., McGee R. X., Newton L. M., Danks A. C., Pottasch S. R., 1983, MNRAS, 204, 53

Simpson J. P., Colgan S. W. J., Rubin R. H., Erickson E. F., Haas M. R., 1995, ApJ, 444, 721

Storey P. J., Hummer D. G., 1995, MNRAS, 272, 41

Storey P. J., Zeippen C. J., 2000, MNRAS, 312, 813

\section{A PPENDIX A：DESCRIPTION OF PIXHUNTER}

This was a 2001 summer project of Aaron Svoboda working at NASA/Ames with RR. There are separate procedures to identify and remove bad pixels from emission lines and from the continuum. For the continuum regions of interest, generally adjacent to emission lines to be measured, sections of the rectified 2D image are identified that are reasonably flat in flux in both the spatial and dispersion directions. Sections are necessary in order to avoid the CS and STIS fiducial bars. PIXHUNTER works by computing the mean pixel value and standard deviation of a given section. After computing these values, it again goes through the image and outputs to a file the coordinates of any pixel whose flux value is further away from the mean than \pm a specified multiple of the standard deviation $(\sigma)$; here we used $5 \sigma$. We linearly interpolate the good data to replace the bad pixel values.

Though the above algorithm for finding the bad pixels works well for the continuum sections, it is incompatible with emission lines, because the flux in an emission line can vary substantially in both the wavelength and spatial dimensions. For cleaning an emission line, PIXHUNTER works interactively with SPLOT. We plot and compare a single column (fixed wavelength) in the line at a time with one of the fitting functions available (e.g. a Legendre polynomial). For each individual column in the line, this fitting function represents the distribution of flux with spatial position along the slit. Following the fit to the emission line distribution, SPLOT will flag all pixels that deviate by more than a specified multiple of $\sigma$ from the functional fit. Various keys/commands permit further processing such as interpolating all of the flagged pixels in a column to the function. When the resulting image is satisfactorily repaired, it can be saved back into the original 2D rectified image. Each column can be treated in this fashion by scrolling through interactively using existing options/tools within SPLOT. There is another method for cleaning bad pixels in the vicinity of pronounced peaks in the spatial distribution. This involves taking the ratio of a single column in the line to the BLKAVG of the entire line. Again, SPLOT is used interactively, operating on each such ratio, in a similar fashion to the first method.

This paper has been typeset from a $\mathrm{T}_{\mathrm{E}} \mathrm{X} / \mathrm{L} \mathrm{T}_{\mathrm{E}} \mathrm{X}$ file prepared by the author. 